\title{
Erratum: Explicitly correlated wave functions of the ground state and the lowest quintuplet state of the carbon atom [Phys. Rev. A 99, 052512 (2019)]
}

\author{
Krzysztof Strasburger
}

(Received 4 June 2019; published 21 June 2019)

DOI: 10.1103/PhysRevA.99.069901

The orbit-orbit magnetic interaction integrals in our work were symmetrized incorrectly. This error was affecting, among others, the wave functions with symmetries used for the considered states of the carbon atom. Correct values of the orbitorbit term and whole relativistic shifts are given in Table I. Consequently, the orbit-orbit energies are converged within a few nanohartrees. In the paragraph before the last paragraph of Sec. III, corrected energies of ${ }^{12} \mathrm{C}$ have to be changed to $E\left({ }^{3} P_{g}\right)=-37.857269$ and $E\left({ }^{5} S_{u}\right)=-37.703662$. Their difference amounts to 0.153607 hartree or $33713 \mathrm{~cm}^{-1}$. The missing contribution amounts to, at least, $6 \mathrm{~cm}^{-1}$. In the last sentence of Sec. III: The orbit-orbit term contributes nearly $16 \mathrm{~cm}^{-1}$ to the excitation energy -almost $15 \%$ of the total contribution of relativistic corrections, amounting to $108 \mathrm{~cm}^{-1}$.

These changes do not affect other conclusions of the paper.

TABLE I. Orbit-orbit term and relativistic shift for ${ }^{\infty} \mathrm{C}$.

\begin{tabular}{|c|c|c|c|c|}
\hline \multirow[b]{2}{*}{ K } & \multicolumn{2}{|c|}{${ }^{3} P_{g}$} & \multicolumn{2}{|c|}{${ }^{5} S_{u}$} \\
\hline & $\left\langle\hat{H}_{4}\right\rangle$ & $\left\langle\hat{H}_{R S}\right\rangle$ & $\left\langle\hat{H}_{4}\right\rangle$ & $\left\langle\hat{H}_{R S}\right\rangle$ \\
\hline 88 & -0.000016296 & -0.013968250 & 0.000056199 & -0.013531324 \\
\hline 129 & -0.000015900 & -0.014003954 & 0.000056148 & -0.013547045 \\
\hline 189 & -0.000015467 & -0.014029658 & 0.000056189 & -0.013561152 \\
\hline 277 & -0.000015362 & -0.014041825 & 0.000056205 & -0.013570427 \\
\hline 406 & -0.000015335 & -0.014053920 & 0.000056184 & -0.013574362 \\
\hline 595 & -0.000015304 & -0.014061628 & 0.000056167 & -0.013578169 \\
\hline 872 & -0.000015264 & -0.014067879 & 0.000056169 & -0.013580168 \\
\hline 1278 & -0.000015230 & -0.014070071 & 0.000056165 & -0.013580265 \\
\hline 1873 & -0.000015222 & -0.014072572 & 0.000056165 & -0.013582685 \\
\hline 2745 & -0.000015209 & -0.014073117 & 0.000056165 & -0.013582669 \\
\hline 4023 & -0.000015203 & -0.014074637 & 0.000056164 & -0.013583055 \\
\hline 5896 & -0.000015200 & -0.014074667 & & \\
\hline
\end{tabular}

